\title{
Fucoidan Induces Apoptosis and Inhibits Proliferation of Hepatocellular Carcinoma via the p38 MAPK/ERK and PI3K/Akt Signal Pathways
}

This article was published in the following Dove Press journal: Cancer Management and Research

\section{Yifei Duan \\ Jingjing $\mathrm{Li}$ \\ Xue Jing \\ Xueli Ding \\ Yanan Yu \\ Qingxi Zhao}

Department of Gastroenterology, The Affiliated Hospital of Qingdao University, Qingdao 266003, People's Republic of China
Correspondence: Qingxi Zhao Department of Gastroenterology, The Affiliated Hospital of Qingdao University, No. 16 Jiangsu Road, Qingdao 266003,

People's Republic of China

$\mathrm{Tel} / \mathrm{Fax}+86-532-82911302$

Email qxzhao007@163.com
Purpose: Fucoidan is a natural bioactive product with broad therapeutic applications. Hepatocellular carcinoma (HCC) is a common malignancy of the liver associated with a relatively high mortality rate; thus, effective treatments are urgently needed. Here, the effects of fucoidan on HCC and the underlying mechanism were explored.

Methods: The proliferation and apoptosis of two HCC cell lines (BEL-7402 and LM3) treated with different concentrations of fucoidan or saline were assessed. The levels of proliferating cell nuclear antigen (PCNA) and CCK8 assay were used to determine proliferative capabilities of BEL-7402 and LM3 cells. Apoptosis of LM3 cells was assessed by Hoechst 33342 staining, Western blotting and flow cytometry. The capability of fucoidan to inhibit the growth of LM3 cells was investigated by monitoring of the p38 MAPK/ERK pathways and the upstream kinases, PI3K/Akt. LM3 xenograft tumors were used for in vivo verification

Results: Cell proliferation and apoptosis assays consistently showed that fucoidan has an inhibitory effect on cell growth. Fucoidan significantly promoted apoptosis of LM3 cells through a mechanism involving activation of caspases 8,9 , and 3 accompanied by changes in B-cell lymphoma-2 (Bcl-2) and Bcl-2-associated X protein (Bax), as well as changes in the phosphorylation of p38 MAPK and ERK. Fucoidan also altered the phosphorylation of its upstream kinase, Akt. Fucoidan treatment markedly reduced the growth of LM3 xenograft tumors, consistent with the in vitro results.

Conclusion: Fucoidan conveys antitumor effects and, thus, should be further explored as a potential treatment option for HCC.

Keywords: fucoidan, carcinoma, hepatocellular, apoptosis, drug therapy

\section{Introduction}

Fucoidan is a fucose-rich sulfated carbohydrate derived from brown seaweed, that possesses anti-oxidant, anti-viral, anti-angiogenic, anti-inflammatory, antiproliferative, and anti-bacterial properties as well as anticancer activities against various tumors, especially those of the digestive system. ${ }^{1-5}$ The mechanisms underlying the anticancer activities of fucoidan may be related to the targeting of multiple signaling molecules and receptors of various cells, including immune and tumor cells, which could directly induce apoptosis and cytotoxicity of cancer cells. ${ }^{6}$

According to recent global cancer statistics, hepatocellular carcinoma (HCC) remains the most common malignancy of the liver. ${ }^{7}$ Even though considerable effort has been devoted to the treatment of $\mathrm{HCC}$, the mortality rate remains 
alarmingly high. ${ }^{8}$ Due to the high frequency of recurrence after surgery and progression after chemoembolization, which underscores the need to identify novel effective adjuvant therapies to improve patient survival. ${ }^{9}$ The pathogenesis of HCC is complex and involves the $\mathrm{Wnt} / \beta$ catenin, mTOR, and MAPK/ERK signaling pathways. ${ }^{10,12}$

Apoptosis is activated through both the mitochondrial (intrinsic) and death receptor (extrinsic) pathways. ${ }^{13}$ The mitochondrial pathway is regulated by the Bcl2 family proteins. ${ }^{14}$ After activation, Bax and Bak undergo homooligomerization and are embedded in the mitochondrial outer membrane, resulting in functional disorders of the membrane. Subsequently, permeabilization of the mitochondrial outer membrane induces the liberation of proapoptotic factors (cytochrome c) to the cytosol, which eventually leads to activation of the caspase cascade. However, this process can be prevented by the antiapoptotic $\mathrm{Bc} 2$ proteins, which can interact with the $\mathrm{BH} 3$ domains of Bax/Bak. ${ }^{15,16} \mathrm{Bcl}-2$ expression was found to be elevated in a variety of tumors, including HCC. ${ }^{17,18}$ Studies have also confirmed the abnormal activation of several pathways associated with apoptosis resistance in HCC, such as the JAK/STAT, RAS/ERK, PI3K/AKT pathways, and the inhibition of p38 MAPK pathway, which promotes apoptosis. ${ }^{19,20}$ Moreover, $\beta$-thujaplicin triggers apoptosis and cell cycle arrest via the p38/ERK MAPK signaling pathway, which causes death of autophagic HepG2 cells via suppression of the Akt/mTOR pathway. ${ }^{21}$ Therefore, it is important to verify the therapeutic effect of drugs for the treatment of HCC by improving sensitivity to apoptosis.

Studies have confirmed that fucoidan can effectively inhibit the replication of the hepatitis $\mathrm{B}$ virus in HepG2.2.15 cells and a mouse model. ${ }^{22}$ Moreover, fucoidan may control HCC metastasis via targeting ITG $\alpha \mathrm{V} \beta 3$ and ID-1. ${ }^{5,23}$ Also, fucoidan can defend primary hepatocytes by resisting bile acid-induced apoptosis and simultaneously prevent the invasive capabilities of $\mathrm{HCC}$ cells by increasing expression of NDRG-1/CAP43. ${ }^{24}$ Fucoidan significantly blocks the proliferation of human hepatoma HLF cells, a poorly differentiated HCC cell line, and maintains the cell cycle in the G1/S phase. ${ }^{2}$ Hence, the hypothesis of the present study is that fucoidan can induce apoptosis and inhibit the proliferation of HCC cells via modulation of the p38 MAPK/ERK and PI3K/Akt pathways. Here, a mouse xenograft tumor model was used to assess the potential of fucoidan as an adjuvant therapeutic agent against $\mathrm{HCC}$.

\section{Materials and Methods}

\section{Reagents}

Fucoidan derived from brown seaweed (Fucus vesiculosus) was obtained from Sigma-Aldrich Corporation ( $\mathrm{St}$ Louis, MO, USA) and stored at $-20^{\circ} \mathrm{C}$ until use. Fucoidan, which is composed of $44.1 \%$ fucose, $31.1 \%$ ash, and $26.3 \%$ sulfate, plus a small amount of aminoglucose, was dissolved in normal saline at a concentration of $10 \mathrm{mg} / \mathrm{mL}$. Primary antibodies against $\beta$-actin, proliferating cell nuclear antigen (PCNA), Bax, Bcl-2, caspase-9, caspase-8, caspase-3, ERK, p-ERK (Thr202/Tyr204), p38 MAPK, p-p38 MAPK, PI3K, Akt and p-Akt were obtained from Cell Signaling Technology, Inc. (Danvers, MA, USA). A Cell Counting Kit-8 (CCK8) was purchased from Dojindo Molecular Technologies, Inc. (Kumamoto, Japan).

\section{Cell Culture}

Two HCC cell lines, BEL-7402 and LM3, were purchased from the Cell Bank of the Chinese Academy of Sciences (Shanghai, China) and maintained in Dulbecco's modified Eagle's medium (Thermo Fisher Scientific, Shanghai, China) supplemented with $100 \mathrm{U} / \mathrm{mL}$ penicillin, $10 \%$ fetal bovine serum (HyClone Laboratories, Inc., South Logan, UT, USA), and $100 \mu \mathrm{g} / \mathrm{mL}$ of streptomycin (Invitrogen Canada Inc., Burlington, ON, Canada) at $37^{\circ} \mathrm{C}$ under an atmosphere of $5 \% \mathrm{CO}_{2} / 95 \%$ air.

\section{Cell Proliferation and Viability}

The BEL-7402 and LM3 cells were seeded in 96-well plates at a concentration of $5 \times 10^{3}$ cells/well. After 1 day of stabilization, the cells were incubated with various concentrations of fucoidan for the indicated times at five replicates at each concentration. Cell proliferation and viability were measured with a CCK 8 kit. Following the addition of CCK8 reagent $(10 \mu \mathrm{g})$, the culture plates were incubated at $37^{\circ} \mathrm{C}$ for $2 \mathrm{~h}$. The absorbance of each well was measured with a Synergy ${ }^{\mathrm{TM}}$ Multi-Mode Microplate Reader (BioTek Instruments, Inc., Winooski, VT, USA) at a wavelength of $450 \mathrm{~nm}$.

\section{Cell Apoptosis Analysis}

LM3 cells at the logarithmic growth phase were seeded into the wells of six-well plates at a density of $1 \times 10^{6}$ cells $/ \mathrm{mL}$ and treated with fucoidan for $48 \mathrm{~h}$. Afterward, the cells were collected, washed with ice-cold phosphate-buffered saline (PBS), and stained with Annexin V-fluorescein 
isothiocyanate and propidium iodide. The proportion of stained cells was analyzed with a BD FACSCanto ${ }^{\mathrm{TM}}$ II flow cytometer (BD BioScience, San Jose, CA, USA).

\section{Hoechst 33342 Staining}

Following treatment with fucoidan for 2 days, the cells were washed with PBS and stained with Hoechst 33342 solution (Sigma Aldrich) ( $1 \mu \mathrm{L}$ to $200 \mu \mathrm{L}$ of PBS). Then, the sixwell plates were maintained at $4^{\circ} \mathrm{C}$ for $20 \mathrm{~min}$ in the dark. The proportion of blue fluorescent cells were examined by fluorescence microscopy (Leica Microsystems, Wetzlar, Germany).

\section{RNA Extraction and Quantitative Real-Time Polymerase Chain Reaction (qRT-PCR)}

Total RNA was extracted from the cells with Trizol reagent (Thermo Fisher Scientific, Waltham, MA, USA) and then reverse-transcribed into complementary DNA using the PrimeScript $^{\mathrm{TM}}$ reverse transcription kit (TaKaRa Biotechnology Co., Ltd., Dalian, China). The gene expression levels of $\mathrm{Bcl} 2$ and Bax were determined by a $7900 \mathrm{HT}$ fast real-time PCR system (Applied Biosystems, Foster City, CA, USA) with primers listed in Table 1.

\section{Western Blot Analysis}

Cellular proteins were extracted with radioimmunoprecipitation assay buffer. Equal amounts of protein $(30 \mu \mathrm{g})$ were separated by $12 \%$ sodium dodecyl sulfatepolyacrylamide gel electrophoresis, then transferred to polyvinylidene fluoride membranes, which were blocked in PBS containing Tween 20 (PBST) with 5\% non-fat milk at room temperature for $60 \mathrm{~min}$. Afterward, the membranes were incubated with primary antibodies overnight at $4^{\circ} \mathrm{C}$. The next days, the membranes were washed with PBST and then incubated with the secondary antibody at room temperature for $60 \mathrm{~min}$. The protein bands were

Table I Nucleotide Sequences of Primers Used for qRT-PCR

\begin{tabular}{|l|l|l|}
\hline Gene & & Primer Sequence (5'-3') \\
\hline Bax & $\begin{array}{l}\text { Forward } \\
\text { Reverse }\end{array}$ & $\begin{array}{l}\text { CCCGAGAGGTCTTTTTCCGAG } \\
\text { CCAGCCCATGATGGTTCTGAT }\end{array}$ \\
\hline Bcl-2 & $\begin{array}{l}\text { Forward } \\
\text { Reverse }\end{array}$ & $\begin{array}{l}\text { GGTGGGGTCATGTGTGTGC } \\
\text { CGGTTCAGGTACTCAGTCATCC }\end{array}$ \\
\hline$\beta$-actin & $\begin{array}{l}\text { Forward } \\
\text { Reverse }\end{array}$ & $\begin{array}{l}\text { CTGGAACGGTGAAGGTGACA } \\
\text { AAGGGACTTCCTGTAACAATGCA }\end{array}$ \\
\hline
\end{tabular}

scanned with the Odyssey ${ }^{\circledR}$ Infrared Imaging System (LI-COR Biosciences, Lincoln, NE, USA).

Animal Experiments and Ethics Statement Six nude mice were housed at a constant temperature of $22^{\circ} \mathrm{C}$ and humidity of $55 \%$ under a $12 \mathrm{~h}$ light-dark cycle, and monitored daily. All animal experiments were conducted in accordance with the National Institutes of Health Guidelines for the Care and Use of Laboratory Animals. The protocols of the animal experiments were approved by the Ethics Committee of the Affiliated Hospital of Qingdao University (Qingdao, China). A mouse xenograft model was established by hypodermic injection of LM3 HCC cells into the upper flank region of each mice at a density of $5 \times 10^{6}(100 \mu \mathrm{L})$. When the area of the subcutaneous tumor tissue reached $1.0 \mathrm{~mm}^{3}$, the mice were assigned to a vehicle (saline) group or a fucoidan treatment group ( $25 \mathrm{mg} / \mathrm{kg} /$ day) ( $\mathrm{n}=3$ mice/group).

Fucoidan was dissolved in physiologic saline for oral administration. Mouse weight and tumor volume were measured every 3 days. Then, the mice were anesthetized and sacrificed by cervical dislocation. Tumor tissues were photographed with the use of a digital camera. Kidneys, liver, lung, spleen and tumor tissue were removed for further analyses.

\section{Hematoxylin and Eosin (HE) Staining}

Fresh tissues were fixed in $4 \%$ paraformaldehyde, embedded in paraffin, and then stored at room temperature. Specimens were sliced to a thickness of $5 \mu \mathrm{m}$, and stained with HE for subsequent experiments. Staining of the cytoplasm and nuclear region was observed under a light microscope.

\section{Immunohistochemical Analysis}

The paraffin-embedded sections were dewaxed in xylene and rehydrated with ethanol. After washing with PBS solution, the microwave restoration method was used for antigen retrieval. The sections were incubated with primary antibodies of Ki-67 (dilution, $1: 100$ ) at $4^{\circ} \mathrm{C}$ overnight and secondary antibodies for $30 \mathrm{~min}$ at $37^{\circ} \mathrm{C}$. Samples were then stained with 3,3'-diaminobenzidine and visualized under a microscope equipped with a digital camera.

\section{Statistical Analyses}

All experimental data are expressed as the mean \pm SD. One-way analysis of variance (ANOVA) and the Student's $t$-test were used to identify significant differences between the control and experimental groups. All experimental data 
were acquired from at least three independent experiments. All statistical analyses were performed with IBM SPSS Statistics for Windows, version 20.0 (IBM Corporation, Armonk, NY, USA). A probability $(p)$ value of $<0.05$ was considered statistically significant.

\section{Results}

\section{Fucoidan Inhibits the Proliferation of HCC Cells}

The effect of fucoidan on the proliferation of HCC cells was examined using the CCK 8 kit. Treatment of LM3 and
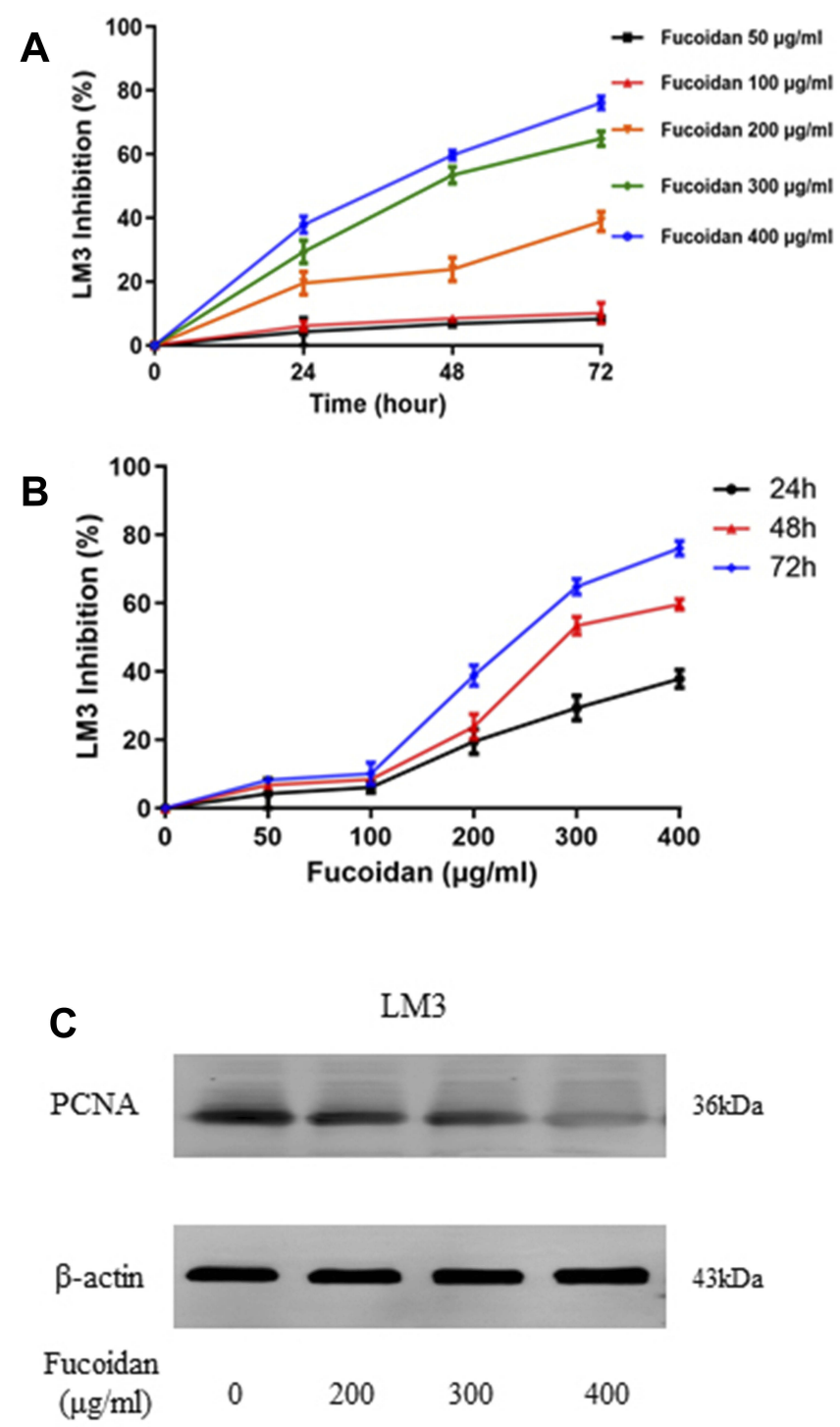

BEL-7402 cells with various concentrations of fucoidan $(50-400 \mu \mathrm{g} / \mathrm{mL})$ for 24,48 , or $72 \mathrm{~h}$ reduced cellular viability in a concentration- and time-dependent manner (Figure 1A and B, respectively). Cell proliferation was inhibited by up to $39 \%, 65 \%$, and $76 \%$ at fucoidan concentrations of 200,300 and $400 \mu \mathrm{g} / \mathrm{mL}$, respectively, for $72 \mathrm{~h}$. PCNA, which was first discovered in the serum of patients with systemic lupus erythematosus in 1978, plays a central role in cellular growth and is an established molecular marker of cell proliferation. ${ }^{25,26}$ Western blot analysis showed that fucoidan downregulated PCNA levels in LM3 and BEL-7402 cells (Figure 1C). These
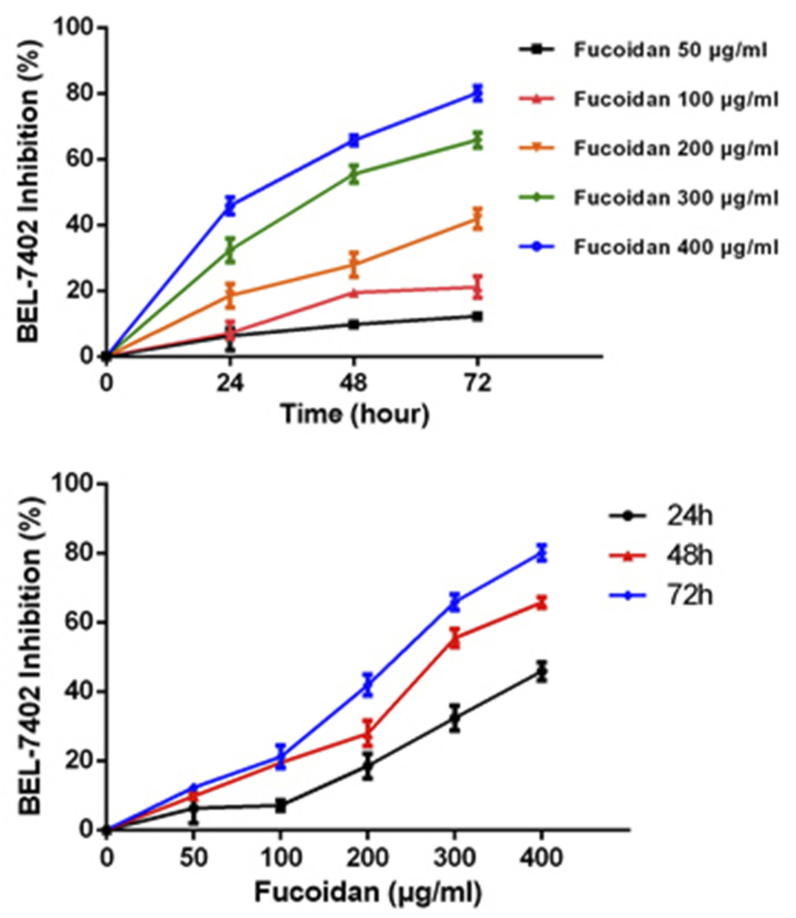

BEL-7402
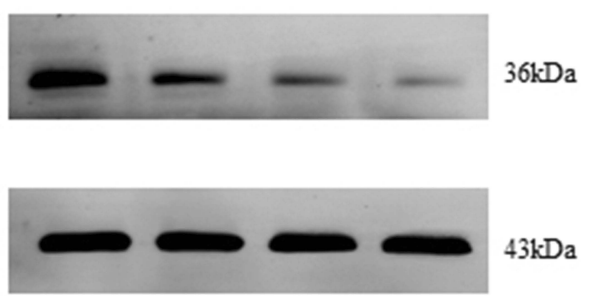

Fucoidan

$(\mu \mathrm{g} / \mathrm{ml}) \quad 0 \quad 200 \quad 300 \quad 400$

Figure I Influences of fucoidan on HCC cell proliferation.

Notes: (A) BEL-7402 and LM3 cells were treated with dd H2O (control) and fucoidan $(50 \mu \mathrm{g} / \mathrm{mL}, 100 \mu \mathrm{g} / \mathrm{mL}, 200 \mu \mathrm{g} / \mathrm{mL}, 300 \mu \mathrm{g} / \mathrm{mL}, 400 \mu \mathrm{g} / \mathrm{mL}$ ) for $72 \mathrm{~h}$. The data showed that fucoidan reduced $\mathrm{HCC}$ cell proliferation in a dose-dependent; (B) BEL-7402 and LM3 cells were treated with dd $\mathrm{H} 2 \mathrm{O}$ (control) and fucoidan (50-400 $\mu$ g/mL) and measured at $24 \mathrm{~h}, 48 \mathrm{~h}$, and $72 \mathrm{~h}$. The statistical data showed that fucoidan lessened HCC cell proliferation in a time-dependent manner; (C) The Western blotting indicated the protein levels of PCNA. 
findings revealed that fucoidan markedly inhibits the growth of HCC cell lines.

\section{Fucoidan Causes Apoptosis of HCC Cells}

LM3 HCC cells were treated with various doses of fucoidan for up to $48 \mathrm{~h}$ and then subjected to Western blotting, flow cytometry, and Hoechst 33342 staining to examine the effects of fucoidan on apoptosis. The results of flow cytometry showed that the percentages of early and late apoptotic cells were significantly greater in the group exposed to fucoidan for $48 \mathrm{~h}$ as compared to the control groups (Figure 2A). Hoechst 33342, which binds to doublestranded DNA, accumulates rapidly in apoptotic cells. Nuclear fragmentation and chromatin condensation, which are characteristic of apoptotic cells, were observed in fucoidan-treated cells stained with Hoechst 33342 reagent, as indicated by bright blue fluorescence (Figure 2A). Caspases are a family of cysteinyl aspartate-specific proteinases that are activated to promote programmed cell death via both the intrinsic and extrinsic pathways, which leads to morphological changes. ${ }^{27,28}$ Western blot analysis showed that fucoidan augmented the levels of active caspase- $3,-9$ and -8 , and cleaved poly (adenosine diphosphate-ribose) polymerase (PARP) in a concentration-dependent manner (Figure 2B).

\section{Fucoidan Alters the Expression Levels of Bcl-2 Family Proteins}

The Bcl-2 family is crucial for modulation of the mitochondrial apoptotic process. ${ }^{29}$ The Bcl-2 family proteins adjust cell death primarily by controlling intracellular proapoptotic and anti-apoptotic signals. ${ }^{14}$ Members of Bcl-2 family proteins include pro-apoptotic BH3-only proteins, pro-apoptotic pore-formers and anti-apoptotic proteins, which are principally involved in the intrinsic pathway of apoptosis. ${ }^{15}$ As compared with the untreated control, fucoidan treatment $(200-400 \mu \mathrm{g} / \mathrm{mL})$ for $48 \mathrm{~h}$ decreased the cytosolic levels of the anti-apoptotic protein Bcl-2 in a dose-dependent manner and augmented the cytosolic levels of the pro-apoptotic protein Bax (Figure 2B). The results of qRT-PCR analysis showed that the mRNA expression levels of Bax and Bcl-2 in LM3 cells assessed by qRT-PCR showed that the mRNA expression levels of Bax increased in LM3 cells, while those of Bcl 2 decreased in a dose-dependent manner (Figure 2C), indicating that fucoidan promoted apoptosis by reducing the ratio of Bcl2 to Bax.

\section{Fucoidan Induces Apoptosis of HCC Cells via the p38 MAPK/ERK and PI3K/ Akt Pathways}

Next, the mechanism underlying the effects of fucoidan on LM3 cells was investigated. Briefly, the effect of fucoidan treatment on the expression levels of MAPKs was investigated to confirm if this signaling pathway participates in regulation of apoptosis. The results showed that fucoidan promoted the phosphorylation of p38 MAPK and reduced the phosphorylation of ERK in a dose-dependent manner (Figure $3 \mathrm{~A}$ and $\mathrm{B}$, respectively). Examination of the upstream pathways demonstrated that fucoidan clearly suppressed the activation of PI3K and inhibited phosphorylation of Akt in a concentration-dependent manner (Figure 3C). These findings demonstrate that fucoidan prevents the proliferation of LM3 cells from possibly by regulation of the p38 MAPK/ERK and PI3K/Akt pathways.

\section{Fucoidan Inhibits the Growth of HCC Cells in Vivo}

To verify the inhibitory effects of fucoidan on the growth of HCC cells in vivo, nude mice were subcutaneously injected with LM3 to generate a xenograft model. After tumor formation, nude mice received a daily dose of fucoidan $(25 \mathrm{mg} / \mathrm{kg})$ or saline. After 3 weeks of feeding, visible changes were observed (Figure 4A). As shown in Figure $4 \mathrm{~B}$, mouse weight and tumor volume increased more rapidly in the vehicle group than in the fucoidantreated group. HE and immunohistochemical staining of Ki-67 were used to examine the level of necrosis and proliferation, respectively. As compared to the salinetreated group, Ki-67 staining showed that the degree of nuclear fragmentation was distinctly reduced in the fucoidan-treated (Figure 4C). Fucoidan was not toxic to the liver, kidney, lung, or spleen tissues (Figure 4D). These data indicate that fucoidan has an inhibitory effect on the proliferation of HCC cells and is not toxic to organisms.

\section{Discussion}

$\mathrm{HCC}$ is associated with high morbidity and mortality rates worldwide. ${ }^{7,30} \mathrm{HCC}$ is generally diagnosed at an advanced stage, thus effective treatment is lacking. Fucoidan continues to attract increasing attention as a natural product for the treatment of HCC and may be less toxic than synthetic drugs. The current study explored the mechanism underlying the anticancer effects of fucoidan to determine 
A

Control
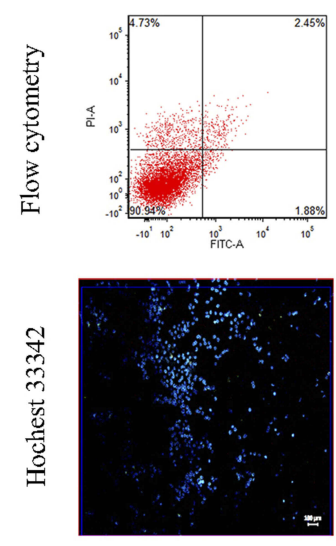

Fucoidan $(200 \mu \mathrm{g} / \mathrm{ml})$
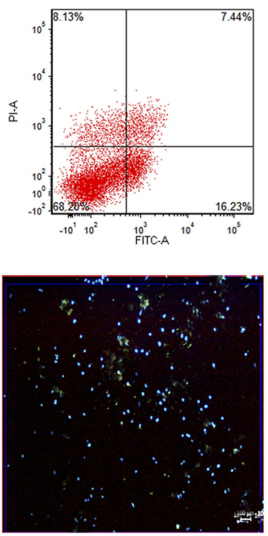

Fucoidan $(300 \mu \mathrm{g} / \mathrm{ml})$

Fucoidan $(400 \mu \mathrm{g} / \mathrm{ml})$
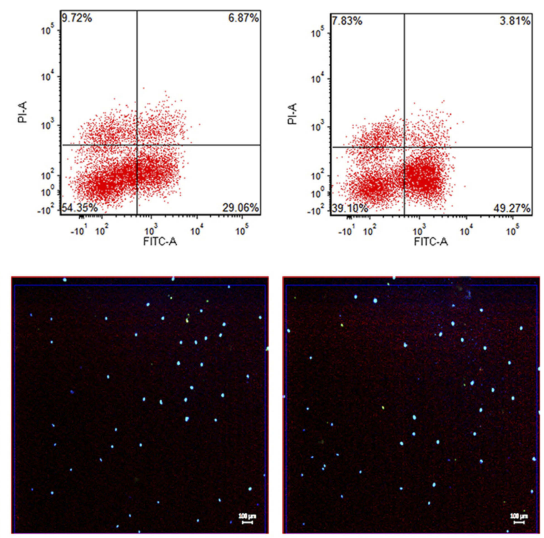

B
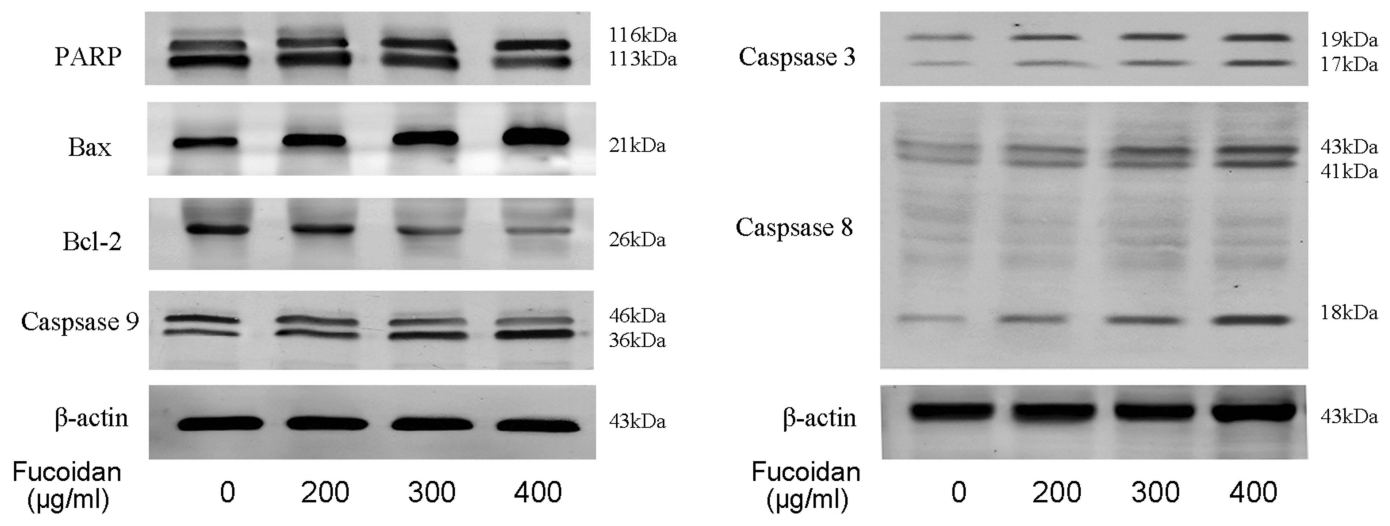

C
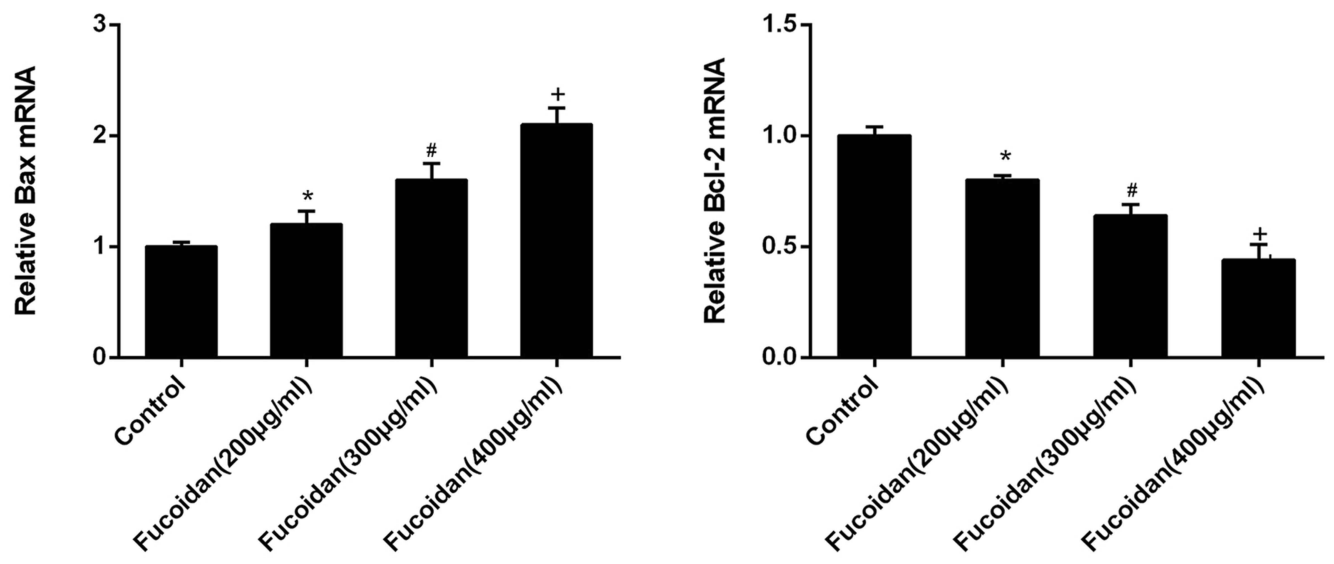

Figure 2 Effects of fucoidan on HCC cell apoptosis.

Notes: (A) LM3 cells were disposed with dd H2O (control) and fucoidan $(200 \mu \mathrm{g} / \mathrm{mL}, 300 \mu \mathrm{g} / \mathrm{mL}, 400 \mu \mathrm{g} / \mathrm{mL})$ for $48 \mathrm{~h}$. Flow cytometry was used to determine the apoptosis of LM3 cells; Nuclear fragmentation of LM3 cells was observed and captured by fluorescence microscopy after fucoidan treatment for $48 \mathrm{~h}$ (Magnification $200 \times$ ); (B) The protein levels of PARP, Bax, Bcl-2, Caspase-3, Caspase-9, and Caspase-8 were determined by Western blotting. (C) The mRNA expression of Bax and Bcl-2 was determined using $\mathrm{qRT}-\mathrm{PCR}\left(\mathrm{n}=3,{ }^{*}, \#,+\mathrm{p}<0.05\right.$ for fucoidan versus control) 
A
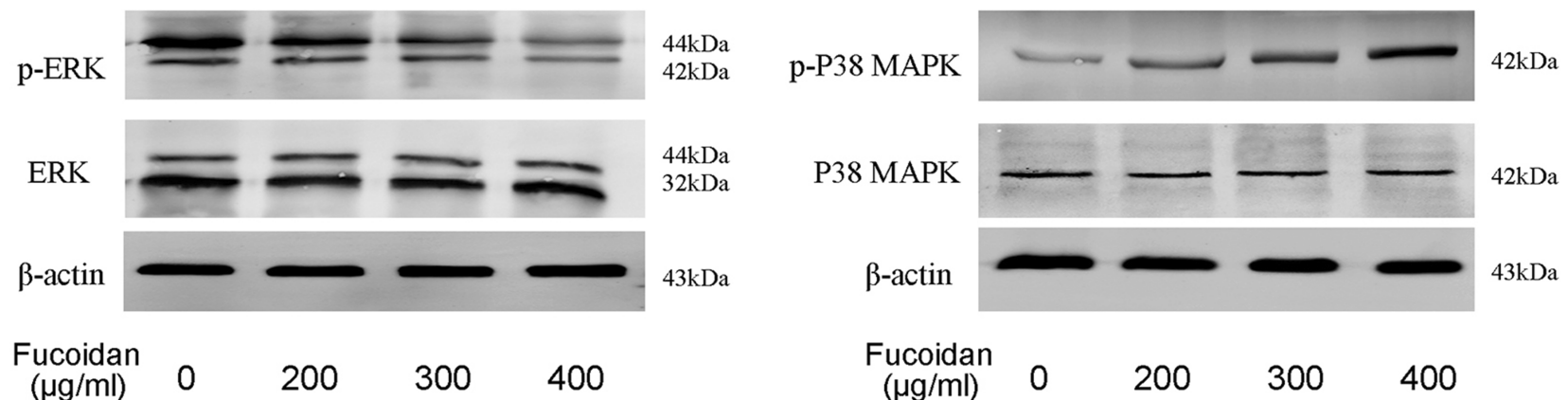

B
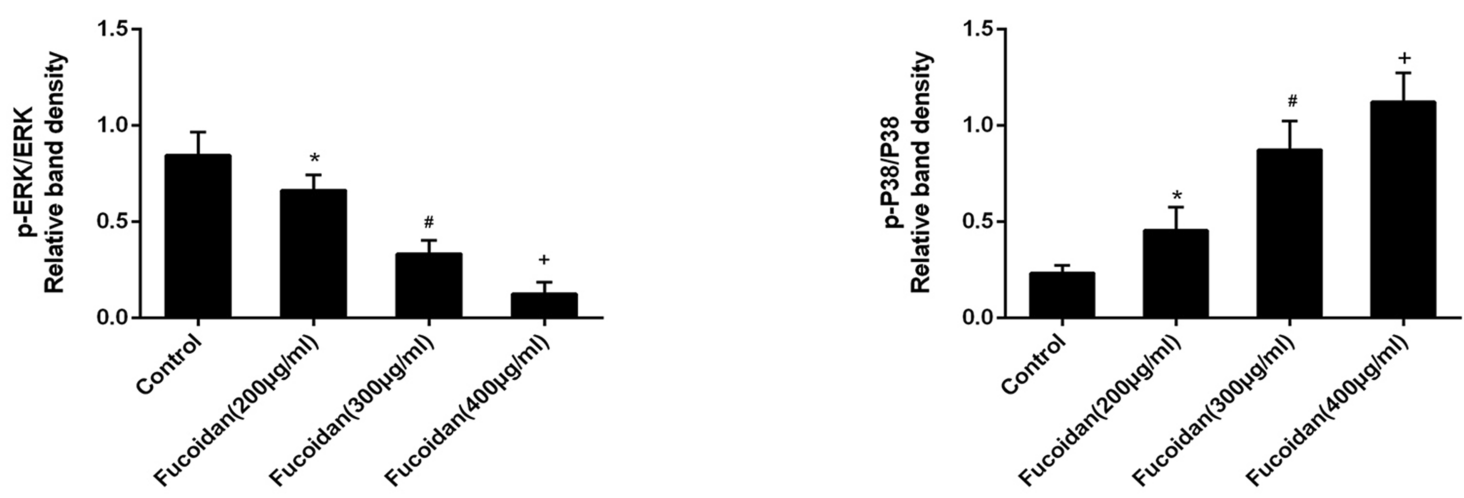

C
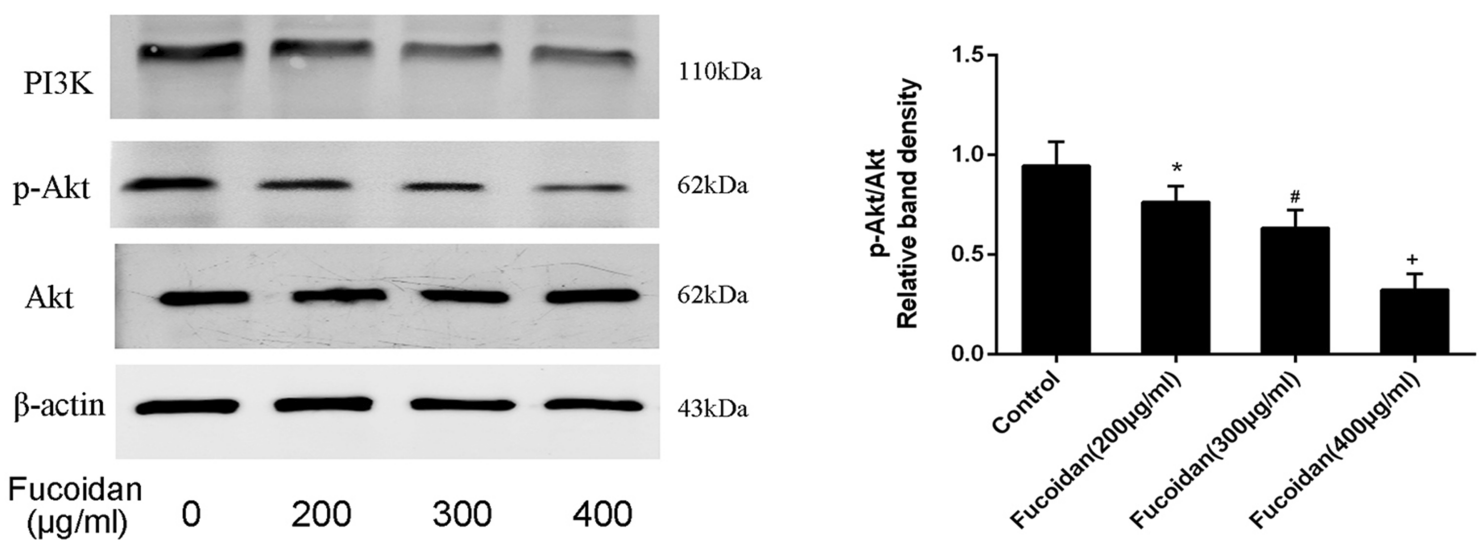

Figure 3 Effects of fucoidan on the PI3K/Akt and P38 MAPK/ERK signaling pathway.

Notes: (A) LM3 cells were treated with dd H2O (control) and fucoidan $(200 \mu \mathrm{g} / \mathrm{mL}, 300 \mu \mathrm{g} / \mathrm{mL}, 400 \mu \mathrm{g} / \mathrm{mL}$ ) for $48 \mathrm{~h}$. The protein levels of ERK, p-ERK, p38 and p-p38 were determined by Western blotting; (B) The ratios of ERK and p-ERK, p38, and p-p38 were calculated using the Odyssey two-color infrared laser imaging system ( $\mathrm{n}=4,{ }^{*}, \#,+\mathrm{p}<$ 0.05 for fucoidan versus control); (C) The protein levels of PI3K, Akt, and $\mathrm{p}$-Akt were determined by Western blotting; the ratios of Akt and $\mathrm{p}$-Akt were evaluated using the Odyssey two-color infrared laser imaging system $\left(n=3,{ }^{*, \#,+} \mathrm{p}<0.05\right.$ for fucoidan versus control).

whether it is suitable treatment option for HCC. The antineoplastic effect of fucoidan was confirmed in vivo using an LM3 xenograft model. The results showed that fucoidan alone exerted a favorable anti-tumor effect in vivo. Moreover, HE staining showed that fucoidan had no obvious toxic effects on the liver, spleen, lung, or kidney of the experimental mice and, thus, should be considered as a relatively safe treatment option.

Cell proliferation is pivotal for the growth and invasive capabilities of HCC cells. Measurement of PCNA mRNA levels and the CCK8 assay were used to comprehensively assess the proliferation of HCC cell lines. 


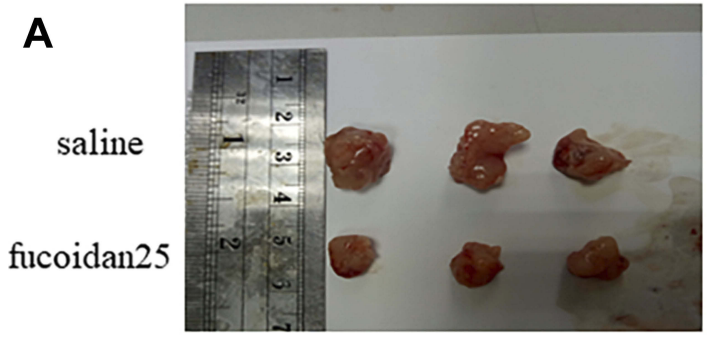

C

$\mathrm{HE}$
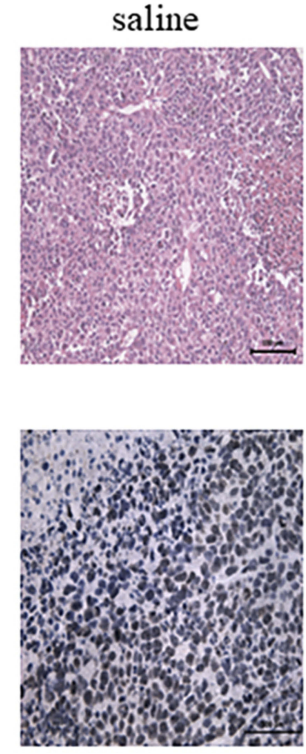

Ki-67
B
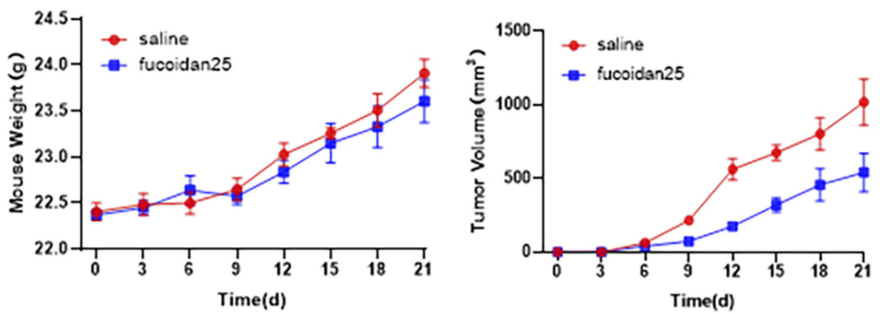

Figure 4 Fucoidan inhibits HCC growth in vivo.

Notes: (A) Morphological images of LM3 cell xenograft tumors in nude mice after 3 weeks of injection. (B) The changes in mouse weight and tumor volume were recorded at the prescriptive time points. (C) Immunohistochemical staining for Ki-67 (magnification 500x) and HE (magnification I00x) indicates the level of necrosis and proliferation. (D) HE staining of liver, lung, spleen and kidney showed no significant changes in the fucoidan-treated group (magnification I00x).

The results showed that fucoidan inhibited the proliferation of LM3 and BEL-7402 cells in a time- and dosedependent manner. Next, the relationship between fucoidan and apoptosis of HCC cells was examined. The distinct morphological changes and increased number of apoptotic LM3 cells, as determined by flow cytometry and Hoechst 33342 staining, were consistent with the decreased Bcl-2 to Bax ratio. The levels of caspases and PARP suggest that fucoidan-induced apoptosis of HCC cells is caspase-dependent and involves both the extrinsic and intrinsic pathways of apoptosis. These results confirmed that fucoidan possesses antiproliferation activities mediated by the induction of apoptotic cell death, as reported previously. ${ }^{31-33}$

The effects of fucoidan are mediated by the PI3K/AKT, ERK, JNK, p38 MAPK, AMPK, GSK and Wnt signaling pathways. ${ }^{6,34}$ Specifically, the PI3K/AKT pathway commonly inhibits apoptosis and promotes cell proliferation, while the MAPK pathway modulates cell proliferation, differentiation, apoptosis, and survival. $^{35}$ MAPKs are a family of serine/threonine kinases that transmit extracellular signals in response to specific intracellular signaling via the ERK, p38 MAPK and JNK pathways. ${ }^{36}$ Activation of the JNK and p38 MAPK is necessary for programmed cell death, and ERKs are related to cancer cell proliferation and resistance to apoptosis. ${ }^{37}$ The p38 MAPK pathway is triggered by environmental stress, inflammatory cytokines, and various mitogens. ${ }^{38}$ Phospho-p38 MAPK not only activates transcription factors in response to different stimuli, but also phosphorylates cytoplasmic proteins, including members of the Bcl family. ${ }^{39}$ The ERK pathway is activated by the small guanine nucleotide-binding protein Ras, which can interact with growth factors and mitogens. After phosphorylation of two residues, ERK1/2 phosphorylates nuclear and cytoplasmic substrates and transactivates transcription factors to enhance cell proliferation and survival. ${ }^{40,41}$ Therefore, these signaling pathways were investigated to gain insight into the mechanism underlying fucoidan-induced apoptosis of $\mathrm{HCC}$ 


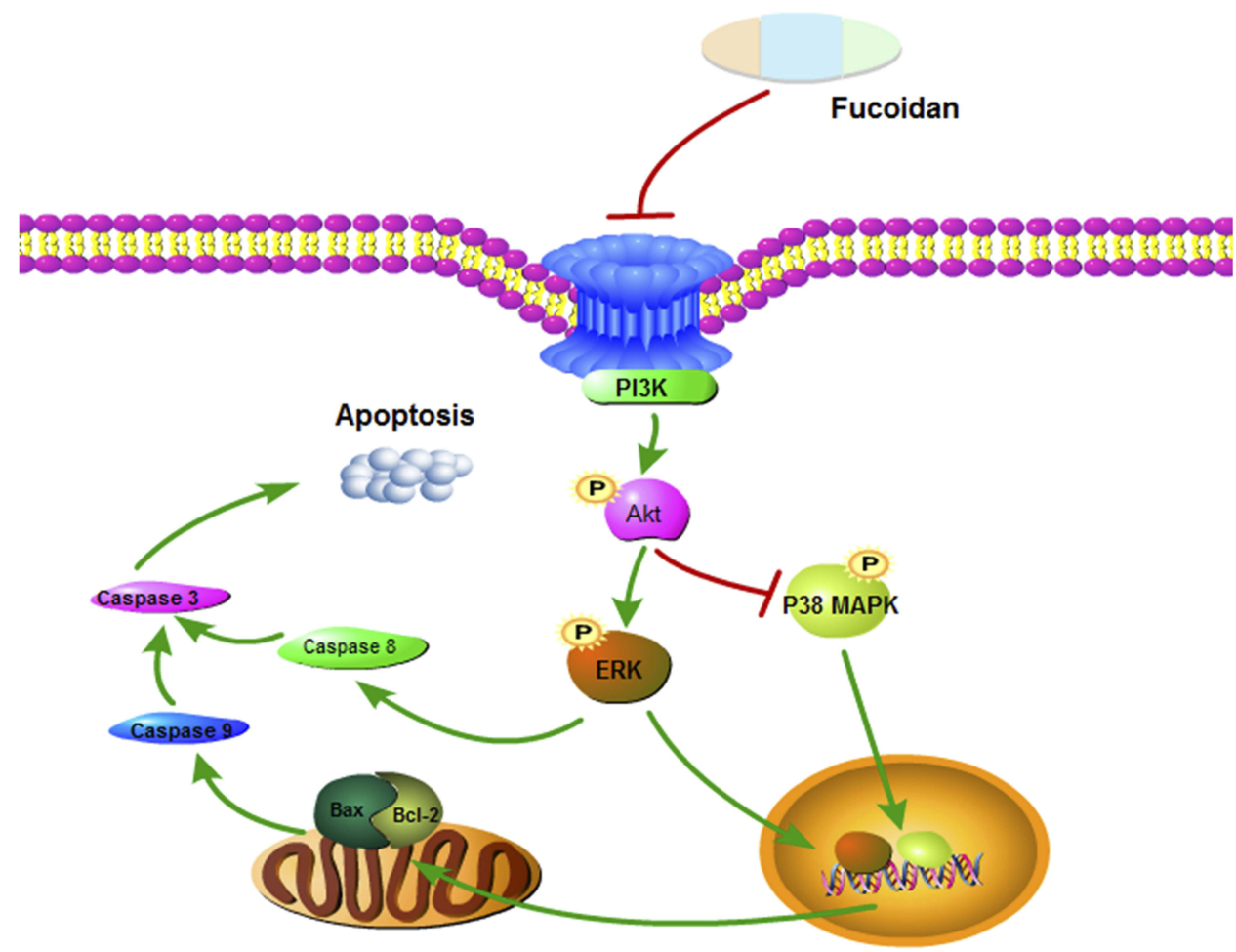

Figure 5 The underlying mechanism of fucoidan action.

Notes: Fucoidan induced the inhibition of PI3K, activation of P38 MAPK and inhibition of ERK lead to decrease in the ratio of Bcl-2 to Bax, which induce mitochondrial dysfunction. The caspases were then activated to induce cell death via mitochondrial apoptosis.

cells. The results of the present study primarily disclosed that the p38 MAPK and ERK pathways are involved in a series of protein kinase cascades and play critical roles in the modulation of fucoidan-induced apoptosis of HCC cells. Fucoidan enhanced the phosphorylation of p38 MAPK and inhibited that of ERK in a dose-dependent manner, suggesting that the p38 MAPK and ERK pathways are crucial for fucoidaninduced apoptosis of HCC cells. Increased phosphorylation of $\mathrm{p} 38 \mathrm{MAPK}$ reduces the expression of $\mathrm{Bcl} 2$ proteins and controls the translocation of Bax from the cytosol to the mitochondria in response to a variety of stimuli, such as caspase 9 and 3, while suppression of the ERK pathway activates Bax, which results in apoptotic cell death. ${ }^{42,43}$

Previous studies have reported a complex crosstalk between the MAPK and PI3K/Akt pathways at different stages of signal transduction. ${ }^{4-46}$ To further elucidate the mechanistic events driving fucoidan-induced apoptosis of HCC, the PI3K/Akt signaling pathway was explored. The results revealed that fucoidan suppresses the PI3K/Akt pathway in HCC cells. These data show that the fucoidan-induced apoptosis of HCC cells occurs via activation of p38 MAPK signaling and inhibition of ERK signaling, which may be suppressed via inactivation of the upstream PI3K/Akt pathways, as a regulatory process. The inactivation of ERK and activation of p38 MAPK decrease the ratio of Bcl-2 to Bax, which results in mitochondrial dysfunction and the release of apoptogenic proteins from the mitochondria to the cytosol, thereby ultimately activating caspases 3 and 9 (Figure 5).

\section{Conclusion}

The results of the present study demonstrated that fucoidan mediates apoptosis through the p38 MAPK/ERK signaling pathways and plays an anti-tumor role in HCC. The underlying mechanism may be dependent on the inhibition of the upstream PI3K/Akt pathway, although further research is needed to confirm these results. The favorable effects of fucoidan suggest its potential as a new anticancer drug for the treatment of HCC.

\section{Acknowledgments}

The research work was financially supported by the Natural Science Foundation of Shandong Province (Nos. ZR201808120020; ZR2016HQ35). This work was also supported by grants from the National Natural Science Foundation of China (Grant No. 81101853) and China Postdoctoral Science Foundation (Grant No. 2019M652332). 


\section{Disclosure}

The authors report no conflicts of interest associated with this work.

\section{References}

1. Luthuli S, Wu S, Cheng Y, Zheng X, Wu M, Tong H. Therapeutic effects of fucoidan: a review on recent studies. Mar Drugs. 2019;17:9. doi:10.3390/md17090487

2. Kawaguchi T, Hayakawa M, Koga H, Torimura T. Effects of fucoidan on proliferation, AMP-activated protein kinase, and downstream metabolism- and cell cycle-associated molecules in poorly differentiated human hepatoma HLF cells. Int $J$ Oncol. 2015;46 (5):2216-2222. doi:10.3892/ijo.2015.2928

3. Etman SM, Elnaggar YSR, Abdallah OY. Fucoidan, a natural biopolymer in cancer combating: from edible algae to nanocarrier tailoring. Int J Biol Macromol. 2019. doi:10.1016/j.ijbiomac.2019.11.191

4. Delma CR, Thirugnanasambandan S, Srinivasan GP, et al. Fucoidan from marine brown algae attenuates pancreatic cancer progression by regulating $\mathrm{p} 53$ - NFkappaB crosstalk. Phytochemistry. 2019;167:112078. doi:10.1016/j.phytochem.2019.112078

5. Pan TJ, Li LX, Zhang JW, et al. Antimetastatic effect of fucoidan-sargassum against liver cancer cell invadopodia formation via targeting integrin alphaVbeta3 and mediating alphaVbeta3/Src/E2F1 signaling. J Cancer. 2019;10(20):4777-4792. doi:10.7150/jca.26740

6. Atashrazm F, Lowenthal RM, Woods GM, Holloway AF, Dickinson JL. Fucoidan and cancer: a multifunctional molecule with anti-tumor potential. Mar Drugs. 2015;13(4):2327-2346. doi: $10.3390 / \mathrm{md} 13042327$

7. Bray F, Ferlay J, Soerjomataram I, Siegel RL, Torre LA, Jemal A. Global cancer statistics 2018: GLOBOCAN estimates of incidence and mortality worldwide for 36 cancers in 185 countries. CA Cancer J Clin. 2018;68(6):394-424. doi:10.3322/caac.v68.6

8. Fong ZV, Tanabe KK. The clinical management of hepatocellular carcinoma in the United States, Europe, and Asia: a comprehensive and evidence-based comparison and review. Cancer. 2014;120 (18):2824-2838. doi:10.1002/cncr.28730

9. Forner A, Reig M, Bruix J. Hepatocellular carcinoma. Lancet. 2018;391(10127):1301-1314. doi:10.1016/S0140-6736(18)30010-2

10. Perugorria MJ, Olaizola P, Labiano I, et al. Wnt-beta-catenin signalling in liver development, health and disease. Nat Rev Gastroenterol Hepatol. 2019;16(2):121-136. doi:10.1038/s41575-018-0075-9

11. Dimri M, Humphries A, Laknaur A, et al. Nqo1 ablation inhibits activation of the PI3K/Akt and MAPK/ERK pathways and blocks metabolic adaptation in hepatocellular carcinoma. Hepatology. 2019:71;549-568.

12. Matter MS, Decaens T, Andersen JB, Thorgeirsson SS. Targeting the mTOR pathway in hepatocellular carcinoma: current state and future trends. J Hepatol. 2014;60(4):855-865. doi:10.1016/j.jhep.2013.11.031

13. Elmore S. Apoptosis: a review of programmed cell death. Toxicol Pathol. 2007;35(4):495-516. doi:10.1080/01926230701320337

14. Ashkenazi A, Fairbrother WJ, Leverson JD, Souers AJ. From basic apoptosis discoveries to advanced selective BCL-2 family inhibitors. Nature Rev Drug Discovery. 2017;16(4):273-284. doi:10.1038/ $\operatorname{nrd} .2016 .253$

15. Kale J, Osterlund EJ, Andrews DW. BCL-2 family proteins: changing partners in the dance towards death. Cell Death Differ. 2018;25 (1):65-80. doi:10.1038/cdd.2017.186

16. Green DR, Llambi F. Cell death signaling. Cold Spring Harb Perspect Biol. 2015;7(12):a006080. doi:10.1101/cshperspect.a006080

17. Correia $\mathrm{C}$, Lee $\mathrm{SH}$, Meng XW, et al. Emerging understanding of Bcl-2 biology: implications for neoplastic progression and treatment. Biochim Biophys Acta. 2015;1853(7):1658-1671. doi:10.1016/j. bbamcr.2015.03.012
18. Marquardt JU, Edlich F. Predisposition to apoptosis in hepatocellular carcinoma: from mechanistic insights to therapeutic strategies. Front Oncol. 2019;9:1421. doi:10.3389/fonc.2019.01421

19. Fabregat I. Dysregulation of apoptosis in hepatocellular carcinoma cells. World J Gastroenterol. 2009;15(5):513-520. doi:10.3748/ wjg. 15.513

20. Wang Y, Sun L, Xia C, Ye L, Wang B. P38MAPK regulates caspase- 3 by binding to caspase- 3 in nucleus of human hepatoma Bel-7402 cells during anti-Fas antibody- and actinomycin D-induced apoptosis. Biomed Pharmacother. 2009;63(5):343-350. doi:10.1016/ j.biopha.2008.05.004

21. Zhang G, He J, Ye X, et al. Beta-Thujaplicin induces autophagic cell death, apoptosis, and cell cycle arrest through ROS-mediated Akt and p38/ERK MAPK signaling in human hepatocellular carcinoma. Cell Death Dis. 2019;10(4):255. doi:10.1038/s41419-019-1492-6

22. Li H, Li J, Tang Y, et al. Fucoidan from Fucus vesiculosus suppresses hepatitis B virus replication by enhancing extracellular signal-regulated Kinase activation. Virol J. 2017;14(1):178. doi:10.1186/s12985-017-0848-8

23. Cho Y, Cho EJ, Lee JH, et al. Fucoidan-induced ID-1 suppression inhibits the in vitro and in vivo invasion of hepatocellular carcinoma cells. Biomed Pharmacother. 2016;83:607-616. doi:10.1016/j. biopha.2016.07.027

24. Cho Y, Yoon JH, Yoo JJ, et al. Fucoidan protects hepatocytes from apoptosis and inhibits invasion of hepatocellular carcinoma by up-regulating p42/44 MAPK-dependent NDRG-1/CAP43. Acta Pharm Sin B. 2015;5(6):544-553. doi:10.1016/j.apsb.2015.09.004

25. Daidoji H, Takasaki Y, Nakane PK. Proliferating cell nuclear antigen (PCNA/cyclin) in plant proliferating cells: immunohistochemical and quantitative analysis using autoantibody and murine monoclonal antibodies to PCNA. Cell Biochem Funct. 1992;10(2):123-132. doi:10.1002/(ISSN)1099-0844

26. Goodlad RA. Quantification of epithelial cell proliferation, cell dynamics, and cell kinetics in vivo. Wiley Interdiscip Rev Dev Biol. 2017;6:4. doi:10.1002/wdev.274

27. Broker LE, Kruyt FA, Giaccone G. Cell death independent of caspases: a review. Clin Cancer Res. 2005;11(9):3155-3162. doi:10.1158/1078-0432.CCR-04-2223

28. Tummers B, Green DR. Caspase-8: regulating life and death. Immunol Rev. 2017;277(1):76-89. doi:10.1111/imr.2017.277.issue-1

29. Banjara S, Suraweera CD, Hinds MG, Kvansakul M. The Bcl-2 family: ancient origins, conserved structures, and divergent mechanisms. Biomolecules. 2020;10:1. doi:10.3390/biom 10010128

30. DeSantis C, Naishadham D, Jemal A. Cancer statistics for African Americans, 2013. CA Cancer J Clin. 2013;63(3):151-166. doi:10.3322/caac. 21173

31. Yang G, Zhang Q, Kong Y, et al. Antitumor activity of fucoidan against diffuse large B cell lymphoma in vitro and in vivo. Acta Biochim Biophys Sin (Shanghai). 2015;47(11):925-931. doi:10.1093/ abbs/gmv094

32. Hsu HY, Lin TY, Hu CH, Shu DTF, Lu MK. Fucoidan upregulates TLR4/CHOP-mediated caspase-3 and PARP activation to enhance cisplatin-induced cytotoxicity in human lung cancer cells. Cancer Lett. 2018;432:112-120. doi:10.1016/j.canlet.2018.05.006

33. Zhu C, Cao R, Zhang SX, Man YN, Wu XZ. Fucoidan inhibits the growth of hepatocellular carcinoma independent of angiogenesis. Evidence-Based Complementary Altern Med. 2013;2013:692549. doi:10.1155/2013/692549

34. van Weelden G, Bobinski M, Okla K, van Weelden WJ, Romano A, Pijnenborg JMA. Fucoidan structure and activity in relation to anti-cancer mechanisms. Mar Drugs. 2019;17:1. doi:10.3390/ md17010032

35. Lamarca A, Mendiola M, Barriuso J. Hepatocellular carcinoma: exploring the impact of ethnicity on molecular biology. Crit Rev Oncol Hematol. 2016;105:65-72. doi:10.1016/j.critrevonc.2016.06.007 
36. Braicu C, Buse M, Busuioc $\mathrm{C}$, et al. A comprehensive review on MAPK: a promising therapeutic target in cancer. Cancers. 2019;11:10. doi:10.3390/cancers 11101618

37. Olson JM, Hallahan AR. p38 MAP kinase: a convergence point in cancer therapy. Trends Mol Med. 2004;10(3):125-129. doi:10.1016/j. molmed.2004.01.007

38. Junttila MR, Li SP, Westermarck J. Phosphatase-mediated crosstalk between MAPK signaling pathways in the regulation of cell survival. FASEB j. 2008;22(4):954-965. doi:10.1096/fj.06-7859rev

39. Cuadrado A, Nebreda AR. Mechanisms and functions of p38 MAPK signalling. Biochem J. 2010;429(3):403-417. doi:10.1042/BJ20100323

40. Roskoski R Jr. Targeting ERK1/2 protein-serine/threonine kinases in human cancers. Pharmacol Res. 2019;142:151-168. doi:10.1016/j. phrs.2019.01.039

41. Cao Z, Liao Q, Su M, Huang K, Jin J, Cao D. AKT and ERK dual inhibitors: the way forward? Cancer Lett. 2019;459:30-40. doi:10.1016/j.canlet.2019.05.025

42. Guo XQ, Cao YL, Zhao L, Zhang X, Yan ZR, Chen WM. p38 mitogen-activated protein kinase gene silencing rescues rat hippocampal neurons from ketamine-induced apoptosis: an in vitro study. Int J Mol Med. 2018;42(3):1401-1410. doi:10.3892/ijmm.2018.3750
43. Farha AK, Dhanya SR, Mangalam SN, Geetha BS, Latha PG, Remani P. Deoxyelephantopin impairs growth of cervical carcinoma SiHa cells and induces apoptosis by targeting multiple molecular signaling pathways. Cell Biol Toxicol. 2014;30(6):331-343. doi:10.1007/s10565-014-9288-z

44. Scheid MP, Duronio V. Dissociation of cytokine-induced phosphorylation of Bad and activation of PKB/akt: involvement of MEK upstream of Bad phosphorylation. Proc Natl Acad Sci USA. 1998;95(13):7439-7444. doi:10.1073/pnas.95.13.7439

45. Aksamitiene E, Kiyatkin A, Kholodenko BN. Cross-talk between mitogenic Ras/MAPK and survival PI3K/Akt pathways: a fine balance. Biochem Soc Trans. 2012;40(1):139-146. doi:10.1042/BST20110609

46. Ho CJ, Gorski SM. Molecular mechanisms underlying autophagy-mediated treatment resistance in cancer. Cancers. 2019;11:11. doi:10.3390/cancers 11111775

\section{Publish your work in this journal}

Cancer Management and Research is an international, peer-reviewed open access journal focusing on cancer research and the optimal use of preventative and integrated treatment interventions to achieve improved outcomes, enhanced survival and quality of life for the cancer patient.
The manuscript management system is completely online and includes a very quick and fair peer-review system, which is all easy to use. Visit http://www.dovepress.com/testimonials.php to read real quotes from published authors. 